\title{
Inhibition of phosphatidylinositol-3-kinase- $\delta$ improves tissue destruction in pemphigoid diseases by impairing neutrophil function
}

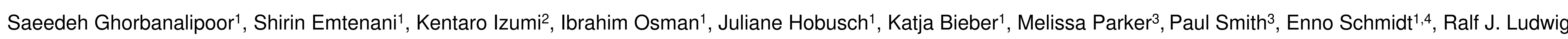

\author{
'Lübeck Institute of Experimental Dermatology (LIED), Lübeck, Germany \\ 2Department of Dermatology, Hokkaido University Graduate School of Medicine, Sapporo, Japan \\ ${ }^{3}$ Incyte Corporation, Wilmington, Delaware, USA
}

${ }^{4}$ Department of Dermatology, University of Lübeck, Lübeck, Germany

\section{Introduction}

Phosphatidylinositol-3-kinase (PI3K) $\delta$-dependent pathways are essential for immune cell activation. Targeting PI3K- $\delta$ has beneficial effect in several preclinical models of inflammatory disorders. PI3K- $\delta$ contributes to T- and B-cell development, survival, and antigen recognition as well as various neutrophil functions. Here, we investigated the effect of INCB050465, a novel PI3K- $\delta$ inhibitor, on disease-driving pathways in the pemphigoid diseases (PDs) namely bullous pemphigoid (BP) and epidermolysis bullosa acquisita (EBA) as autoantibody-induced and neutrophil-dependent diseases.
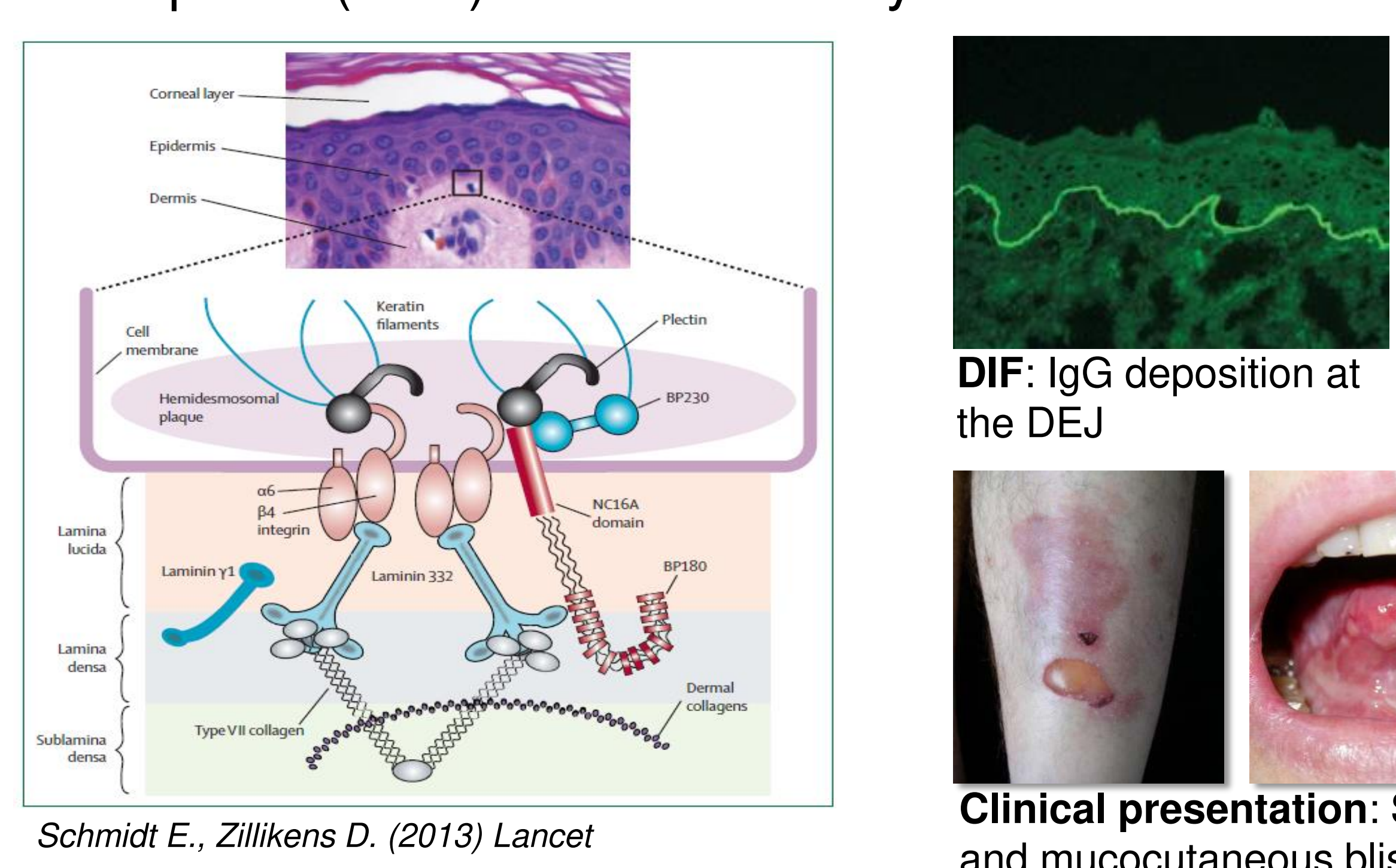
DIF: IgG
the DEJ

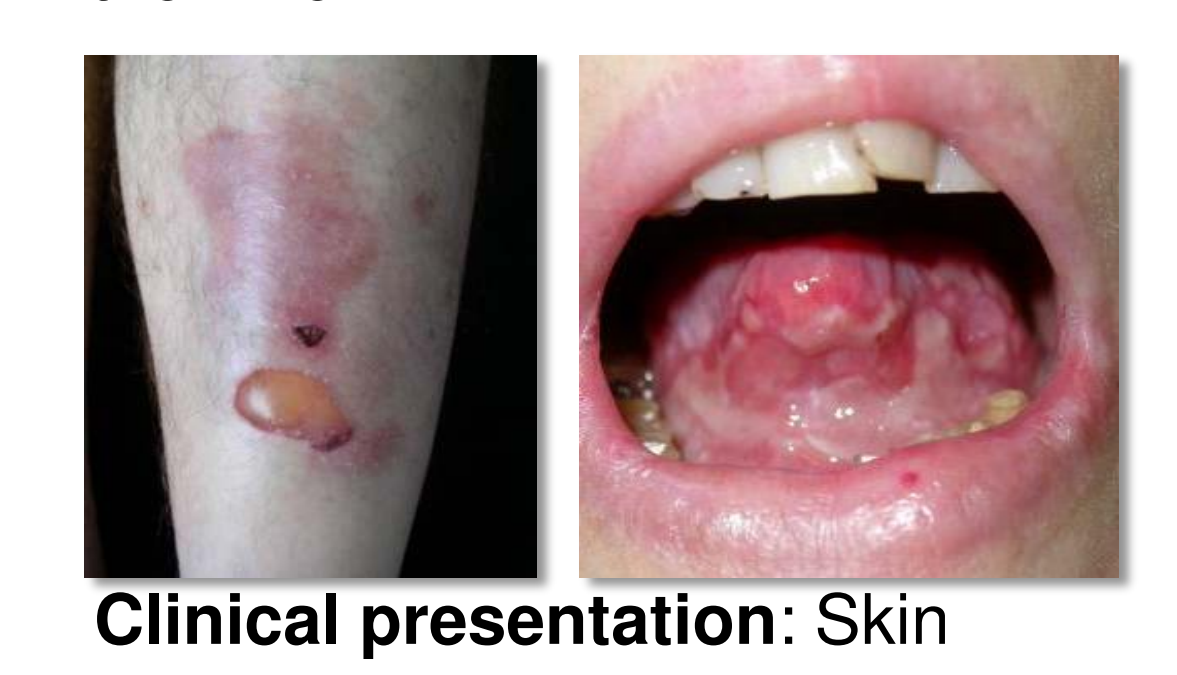

Methods

\section{In vitro study}

- ROS release assay: It is a luminol-amplified chemiluminescence technique which models the immune complex (IC)-induced PMN activation in PDs. Upon stimulation of PMNs using ICs, consisting of recombinant human-NC16A antigen/murine COL7 antigen and BP patient $\mathrm{lgG} /$ anti-murine $\mathrm{lgG}$, they generate ROS which lead to oxidation of luminol and emission of blue luminescence that is detectable by a luminometer.

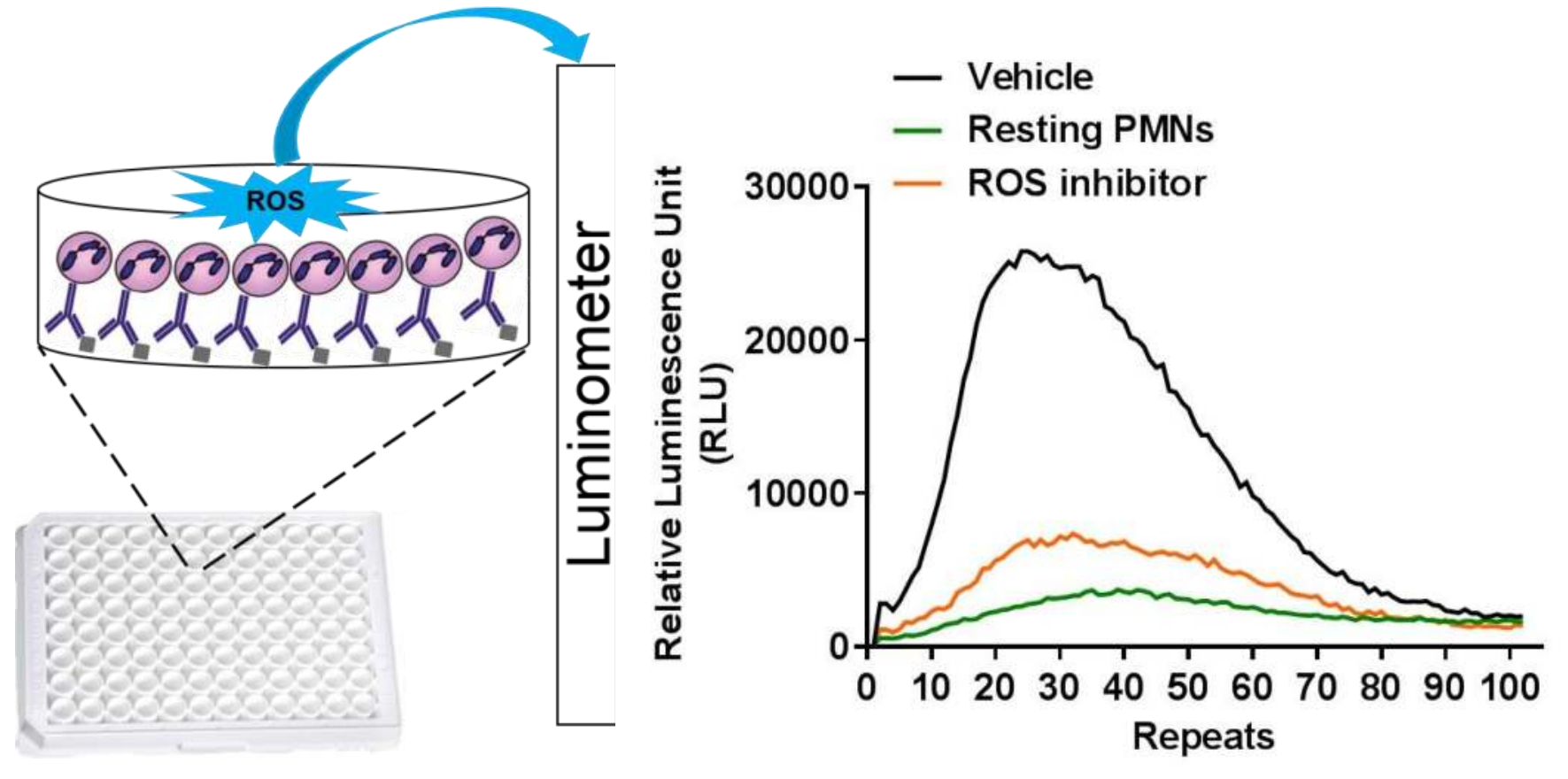

Cell-free ROS release assay: This system comprises glucose/glucose oxidase (G/GOX), catalase (CAT), and myeloperoxidase (MPO) that enables regulated generation of hypochlorous acid $(\mathrm{HClO})$ and $\mathrm{H}_{2} \mathrm{O}_{2}$ that simulates the oxidative burst of neutrophils.

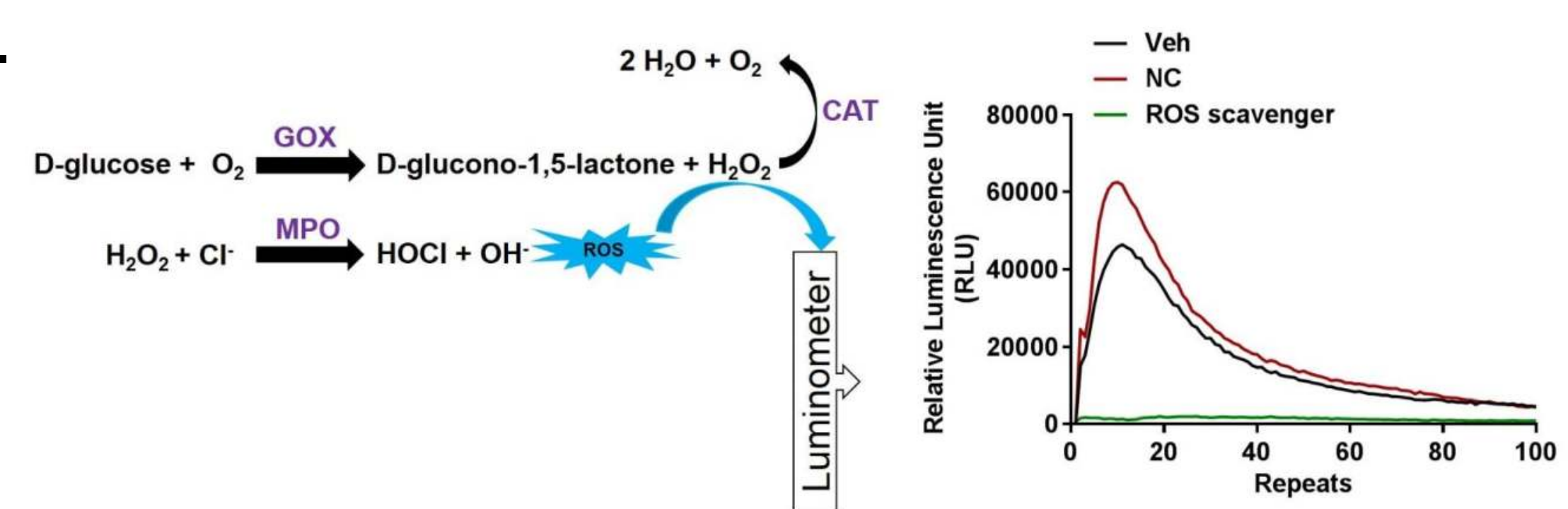

Cryosection assay: An approach that mimics PMN-induced tissue damage (split formation) in PDs.

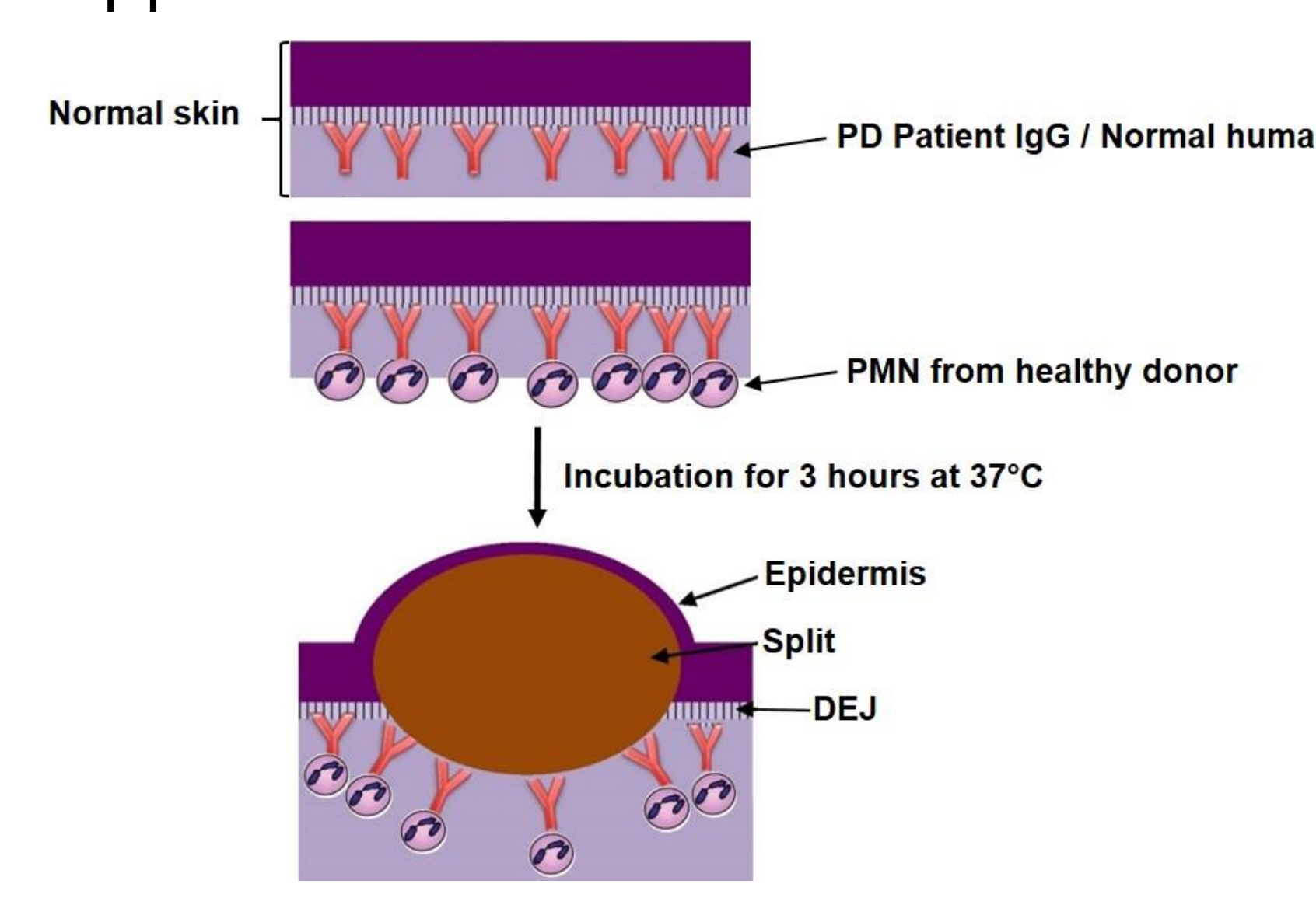

Cytotoxicity assay: a flow cytometry-based assay using Annexin V (AV) and propidium iodide $(\mathrm{PI})$ for detection of viable, early and late apoptotic cells.

\section{In vivo study}

- Immunization-induced EBA: B6.s mice were immunized with 120 $\mathrm{gg} / \mathrm{head}$ vWFA2 protein (immunodominant domain of murine type VII collagen) and Titermax as an adjuvant. When percentage of affected body surface area reached $>2$, mice were randomly allocated to treatment either with drug or vehicle $(5 \%(\mathrm{v} / \mathrm{v}) \quad \mathrm{N}, \mathrm{N}$ Dimethylacetamide (DMA) in methylcellulose $(0.5 \%)$ ). Methylprednisolone (MP) was utilized as a reference treatment.
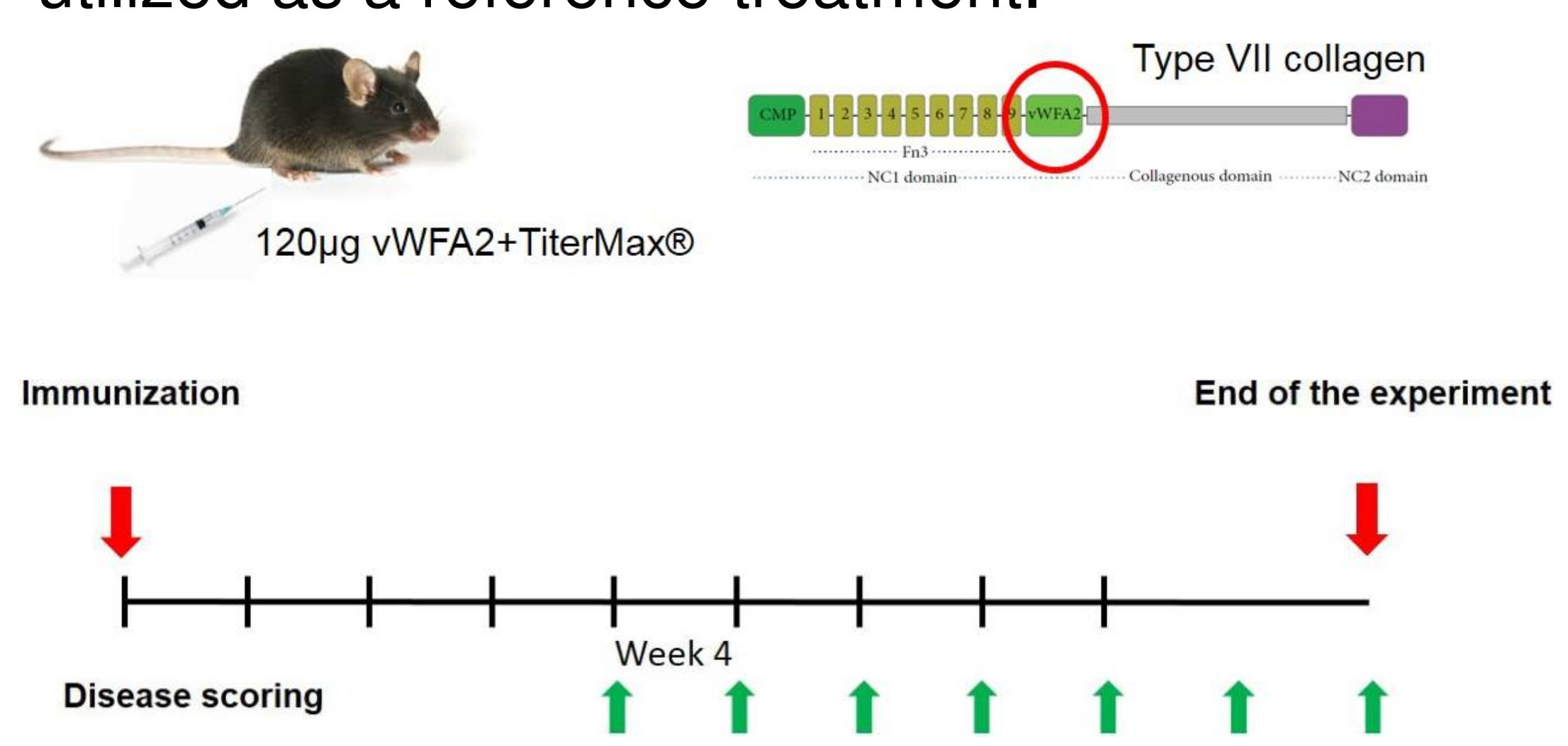

\section{Results}

- PI3K- $\delta$ inhibition decreases ROS generation by IC-activated PMNs.
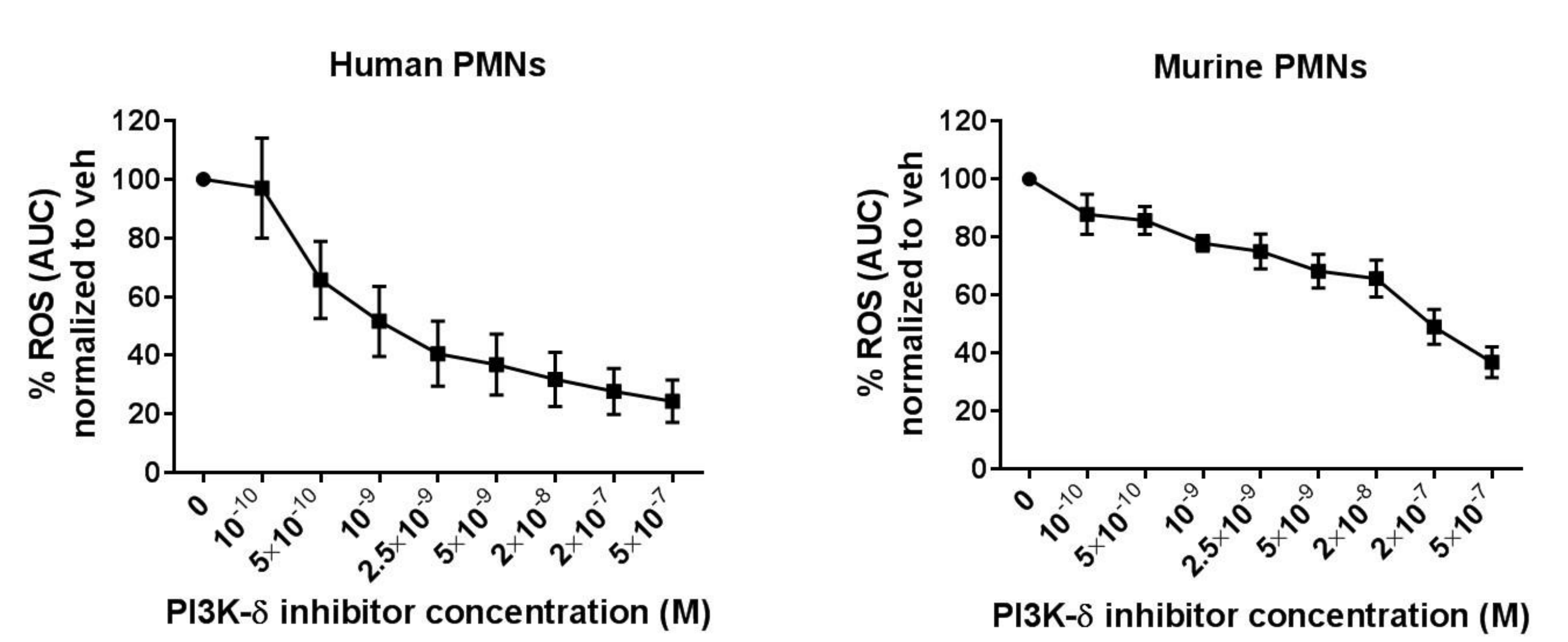

- ROS-inhibition of INCB050465 was not attributed to its antioxidant activity.

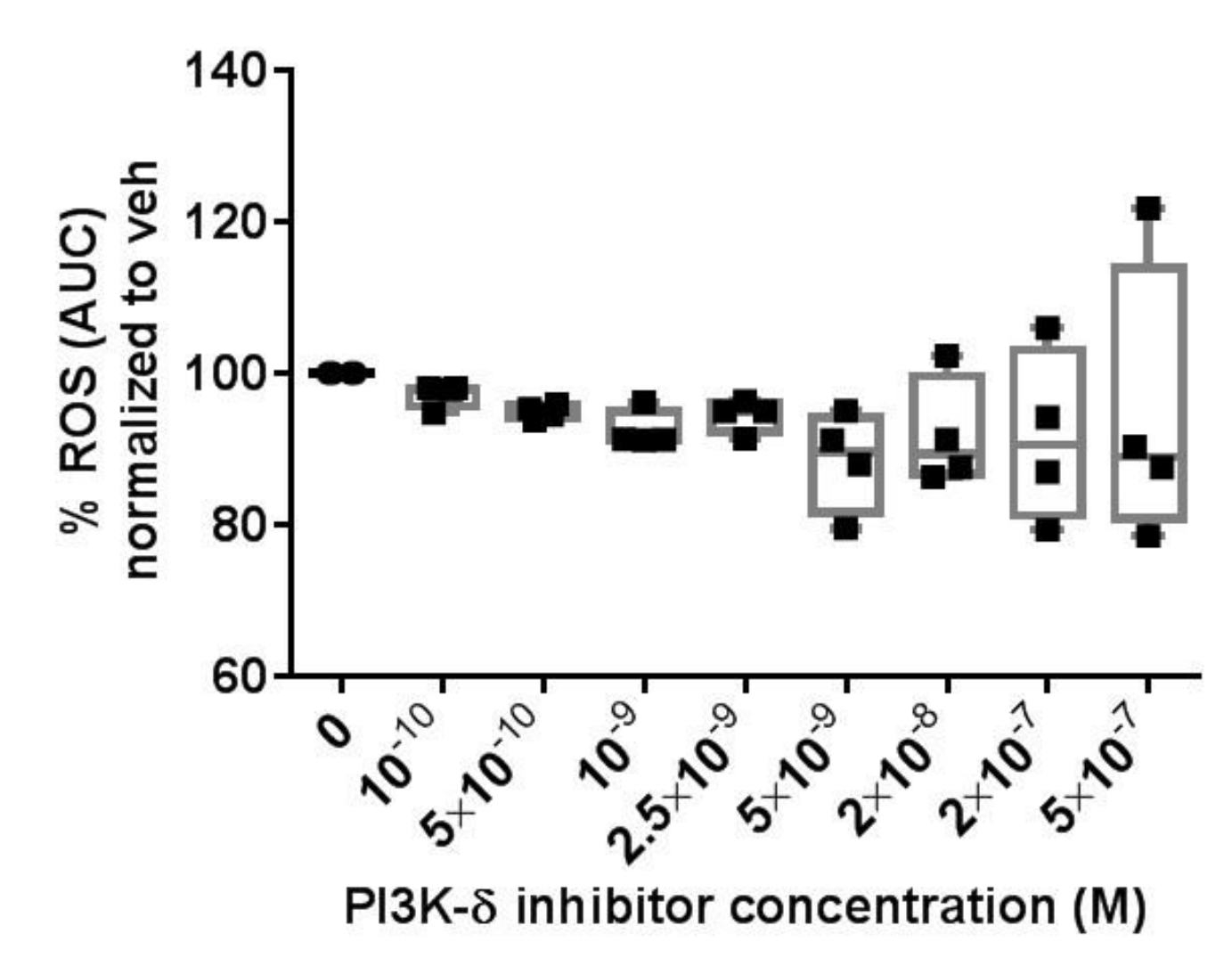

- Split formation is hampered by PI3K- $\delta$ inhibition in a dose dependent manner.

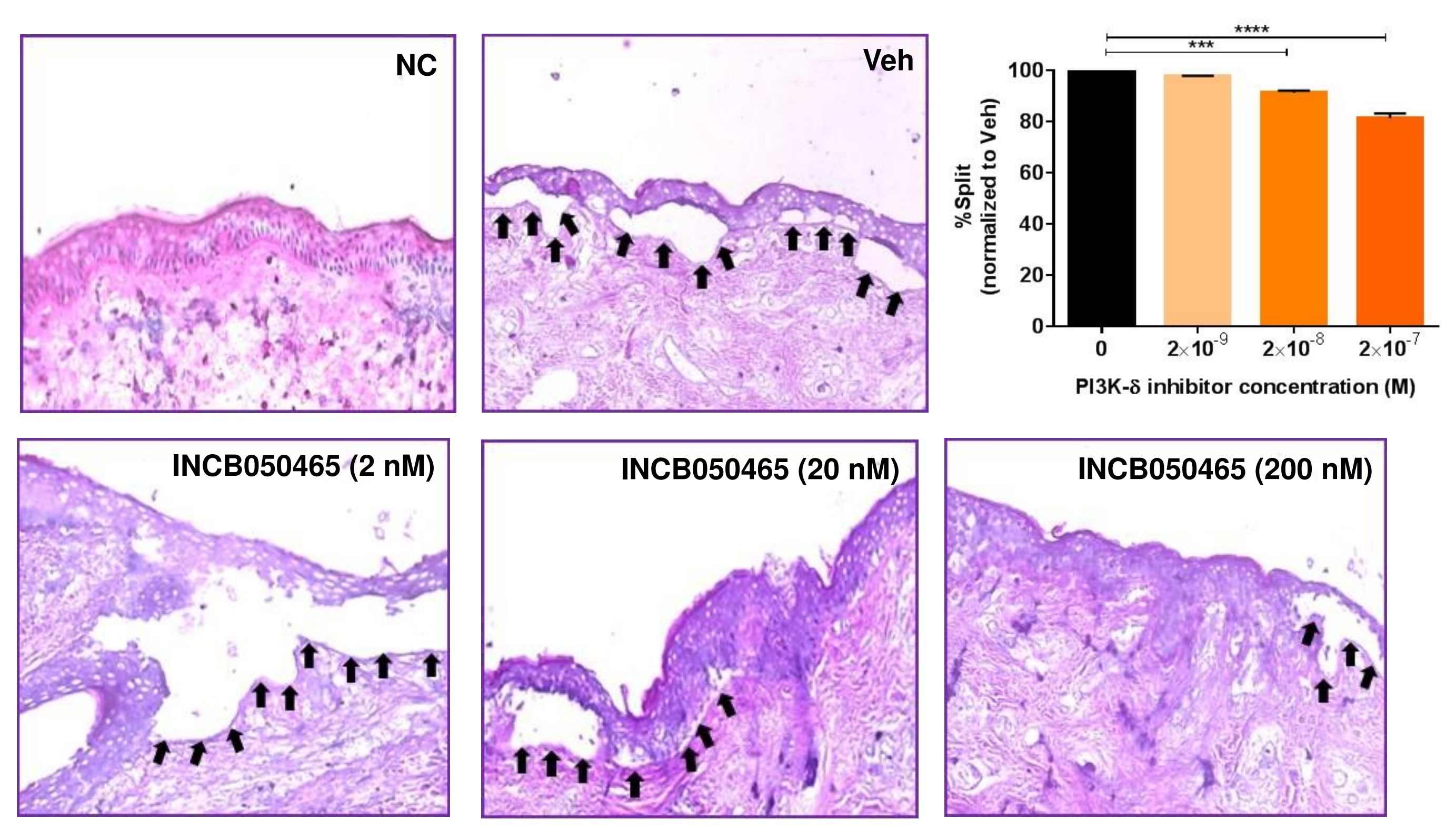

- INCB050465 demonstrated no drug-induced toxicity in PMNs.
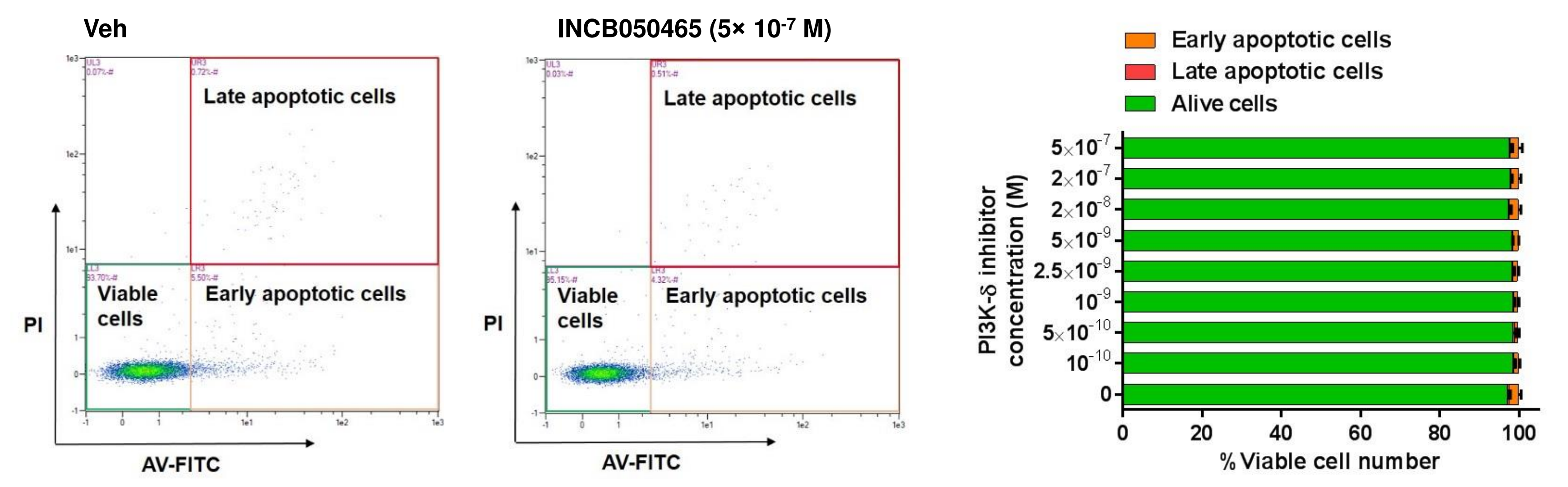

- PI3K- $\delta$ inhibition hinders disease progression in established immunization-induced EBA.
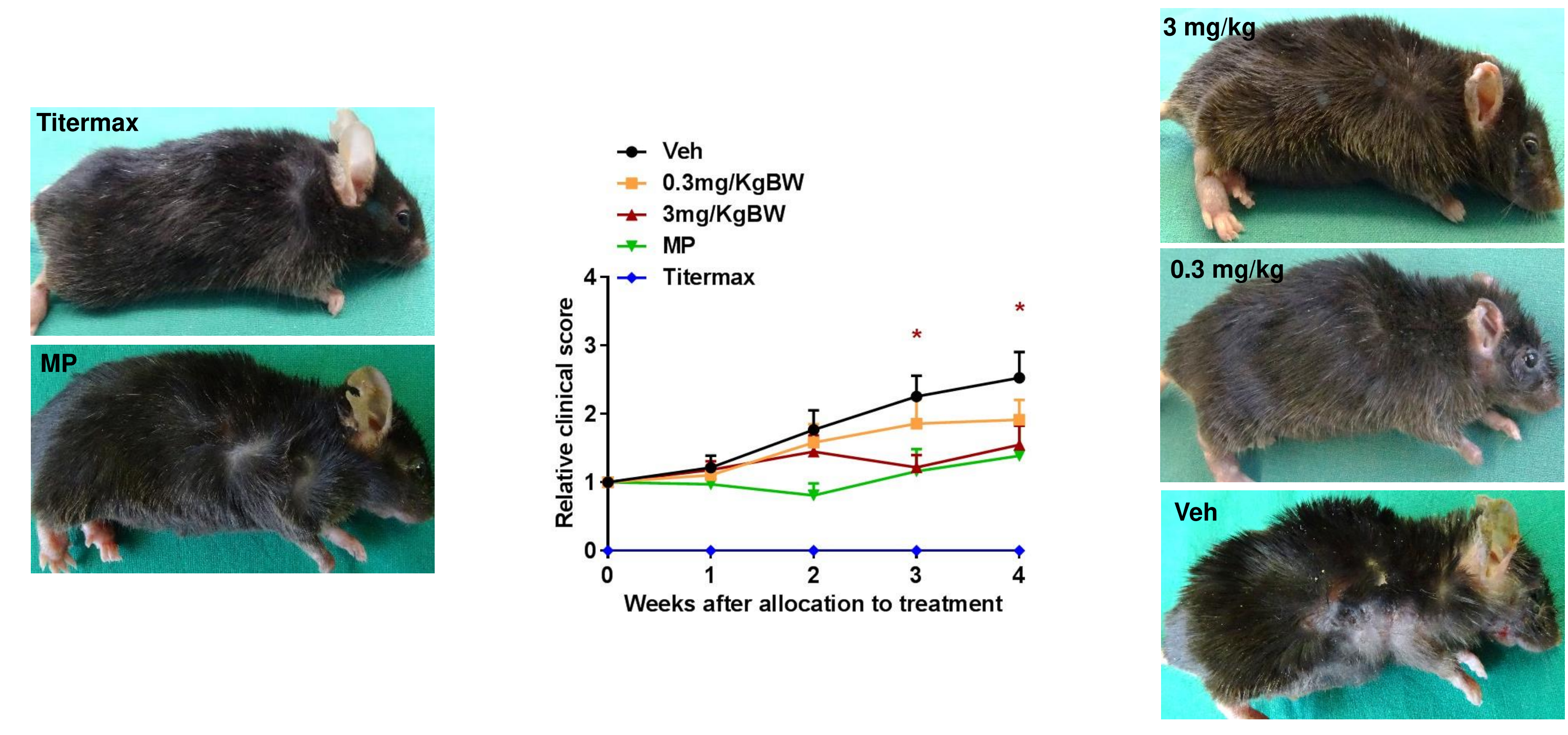

\section{Conclusion}

PI3K-ס inhibition led to:

- impairment of ROS generation by IC-stimulated PMNs.

- diminution of Ab-mediated tissue damage by PMNs.

- reduction of disease progression in established immunization-induced EBA. 